\title{
EFECTO DE DIFERENTES PROPORCIONES DE NITRÓGENO/ FÓSFORO EN EL CRECIMIENTO Y TOXICIDAD DE ALEXANDRIUM CATENELLA (DINOFLAGELLATA)
}

\author{
EFFECT OF DIFFERENT NITROGEN/PHOSPHORUS NUTRIENT RATIOS ON GROWTH \\ AND TOXIN CONTENT OF ALEXANDRIUM CATENELLA (DINOFLAGELLATA)
}

\author{
Cristián Garrido", Máximo Frangópulos" \& Daniel Varela"***
}

\begin{abstract}
Among the environmental factors that control the formation of toxic blooms in phytoplanktonic species, nutrients are considered fundamental. Alexandrium catenella strain AY2 was cultured during one month, in order to determine the specific growth rate, abundance and toxicity in different nutrients ratios. The three conditions studied were: nitrogen-saturated medium ( $\mathrm{N}: \mathrm{P}=24: 1$ ), Nitrogen-balanced $(\mathrm{N}: \mathrm{P}=12: 1)$ and Nitrogen limited medium ( $\mathrm{N}: \mathrm{P}=3: 1$ ). Phosphorus was considered limiting in all three scenarios. Two parallel experiments were conducted, the first one to assess cellular counts every three days and the other to determine the cell toxicity, which were measured every six days. Our results showed that the fastest specific growth rate was in the nitrogen-saturated medium with $0.49 \mu$, but the highest abundance (in the exponential phase) occurred in the in the nitrogen-balanced medium with a value of 5,266 cells $\mathrm{ml}^{-1}$ at day 21 , while the lowest abundance was found in the nitrogen-limited medium, on day 12 , with 4,072 cells $\mathrm{ml}^{-1}$. Regarding toxicity, the highest value was observed on day 12 with $24.56 \mathrm{fmol}$ cell $^{-1}$ in the nitrogen-limited treatment and the lowest showed values of $8.85 \mathrm{fmol} \mathrm{cell}^{-1}$ in the nitrogenbalanced medium. The GTX3 toxin was dominant, being found in the 3 treatments. The results in this study suggest that the nitrogen/phosphorus ratios influenced in a non-synergistic way in the growth and toxin production of $A$. catenella, resulting into a stimulation of growth by nitrogen (ratios $24: 1$ and 3:1) and toxins increased by limitation of this nutrient.
\end{abstract}

Key words: Dinoflagellate, Alexandrium catenella, nutrients, growth, toxins.

1 Instituto de Fomento Pesquero, base zonal Punta Arenas, Enrique Abello 0552 Punta Arenas, Chile. cristián.garrido@ifop.cl 2 Centro Regional de Estudios del Cuaternario de Fuego-Patagonia y Antártica (Fundación CEQUA); Av. 21 de Mayo 1690, Casilla 737, Punta Arenas, Chile.

3 Centro de Investigación I-mar, Universidad de los Lagos, Casilla 557, Puerto Montt, Chile. 


\section{RESUMEN}

Entre los factores ambientales que controlan el desarrollo de floraciones en especies fitoplantónicas tóxicas, los nutrientes son considerados como fundamentales. La cepa AY2 de Alexandrium catenella fue cultivada durante un mes, con la finalidad de determinar su tasa de crecimiento específica, abundancia $y$ toxicidad en diferentes proporciones de nutrientes. Las tres condiciones estudiadas fueron: medio de Nitrógeno-saturado ( $\mathrm{N}: \mathrm{P}=24: 1$ ), Nitrógeno-equilibrado ( $\mathrm{N}: \mathrm{P}=12: 1$ ) y Nitrógeno-limitante ( $\mathrm{N}: \mathrm{P}=3: 1$ ). El fósforo se consideró limitante en los tres escenarios. Dos experimentos paralelos fueron realizados, el primero para evaluar los conteos celulares cada tres días y el otro para determinar la toxicidad en las células, las cuales se midieron cada seis días. Nuestros resultados mostraron que la tasa de crecimiento específica más rápida fue observada en la proporción de nitrógeno-saturado con $0,49 \mu$, pero la abundancia máxima (en la fase exponencial) se registró en el medio de nitrógeno-equilibrado, con un valor de 5266 células $\mathrm{ml}^{-1}$ en el día 21, mientras que la menor abundancia se encontró en la proporción de nitrógeno-limitante, en el día 12, con 4.072 células $\mathrm{ml}^{-1}$. En cuanto al contenido tóxico, el valor más alto se observó en el día 12 con 24,56 fmol cél ${ }^{-1}$ en el tratamiento nitrógeno-limitante y el menor tuvo valores de 8,85 $\mathrm{fmol} \mathrm{cel}^{-1}$ en el medio nitrógeno-equilibrado. La toxina GTX3 fue la toxina dominante, encontrándose en los 3 tratamientos. Los resultados permiten concluir que para el presente estudio las proporciones nitrógeno/fósforo influyeron de forma no sinérgica en el crecimiento y la producción de toxinas en $A$. catenella, lo que se traduce en una estimulación del crecimiento por parte del nitrógeno (proporciones 24:1 y 3:1) y en un aumento de toxinas por limitación de este nutriente.

Palabras clave: Dinoflagelado, Alexandrium catenella, nutrientes, crecimiento, toxinas.

\section{INTRODUCCIÓN}

El género Alexandrium es asociado a eventos recurrentes de toxinas y floraciones de algas nocivas (FAN) a nivel mundial (Cembella 1998), esto último se ha convertido en un problema transversal de nuestro planeta que afecta e impacta diferentes ámbitos del la actividad humana. En las últimas décadas las FAN han experimentado un fuerte incremento en su frecuencia, duración, intensidad y cobertura geográfica (Hallegraeff, 1995, Glibert et al. 2005), fenómeno que no ha estado ajeno a las costas Chilenas por parte de Alexandrium catenella

A. catenella está ampliamente distribuido en el mundo (Lilly et al. 2007) y en aguas marinas de Chile ha presentado varios eventos de floraciones, los que se han concentrado principalmente en las zonas más australes (Los Lagos, Aysén y Magallanes) donde las toxinas paralizantes producidas por este organismo han causado cuantiosos impactos en la pesca, acuicultura y salud pública. A pesar de haber sido detectada su presencia en aguas de la región de Magallanes en 1972 (Guzmán et al. 2002), numerosos aspectos de la biología y ecología de este dinoflagelado aún son desconocidos.
Hasta hace no mucho tiempo se pensaba que A. catenella (Whedon \& Kofoid, 1936; Balech, 1985) y otros dinoflagelados eran autótrofos obligados, pero recientemente se ha demostrado que poseen al menos dos modos mas de alimentación (Jeong et al. 2010), en el caso particular de A. catenella, está comprobado que no solo es autótrofo sino que también heterótrofo (Jeong et al. 2005, Seong et al. 2006), lo que lo convertiría en mixotrófico (Burkholder et al. 2008).

Existen muchos tipos de toxinas asociadas a este organismo, siendo la principal y la más letal para humanos la saxitoxina (Shimizu, 1996). Actualmente los estudios llevados a cabo en laboratorio con distintas especies de Alexandrium y distintas proporciones de nutrientes nos entregan resultados sobre los mecanismos que generan sus toxinas $y$ el tipo de composición de las mismas, revelando que no existe un patrón, sino que la variabilidad es amplia (Bechemin et al. 1999, Collos et al. 2004, Chee et al. 2004, Choua et al. 2004, Leong et al. 2004, Lim \& Ogata 2005, Maestrini et al. 1999, Navarro et al. 2006 y Wang et al. 2005).

En cuanto a sus tasas de crecimiento, Alexandrium muestra diferencias, dependiendo de las 
condiciones ambientales en las que se encuentre; en este ámbito nuevamente los nutrientes como el nitrógeno y fósforo juegan un rol clave (Collos et al.2004; 2007; 2009; Jauzein et al. 2010). Estos nutrientes afectan de diversas maneras el crecimiento y la síntesis de toxinas en los dinoflagelados. Junto a lo anterior, diferentes proporciones de estos nutrientes puede tener un efecto distinto entre cepas de la misma especie, (Boyer et al. 1987, Anderson et al. 1990, Bechemin et al. 1999, John \& Flynn 2000 y Wang et al. 2005). También, diversos autores han observado que limitación de nitrógeno y fósforo induce un aumento en la cantidad de toxinas, como también lo hace solo el fósforo cuando se encuentra en una concentración limitante (Johansson \& Graneli 1999, Choua et al. 2004, Hwang \& Lu 2000, Lim et al. 2006, Maestrini et al. 1999 y Murata et al. 2006).

Actividades humanas como la eutrofización, pueden favorecer el crecimiento de los dinoflagelados, ya que ingresan nutrientes al sistema, y éstos pueden conducir a una gran biomasa fitoplanctónica. Pero algunos dinoflagelados prefieren ciertas formas químicas de nutrientes en vez de otras (Anderson et al. 2002).

El presente estudio pretende dilucidar como es el crecimiento y toxicidad del dinoflagelado tóxico A. catenella a diferentes concentraciones de nitrato, fosfato y a su vez como la variación de éstos genera cambios en la composición y concentración de toxinas.

\section{MATERIALES Y MÉTODOS}

La cepa AY2 de Alexandrium catenella, aislada en la región de Aysén, fue cultivada en medio L1, sin silicatos (Guillard \& Ryther 1993), con agua de mar previamente filtrada por un filtro de fibra de vidrio GF/F Whatman de 0,2 $\mu \mathrm{m}$ de poro, a la cual posteriormente se procedió a disminuirle la salinidad a 30 PSU, utilizando agua destilada; finalmente fue llevada al autoclave a $120^{\circ} \mathrm{C}$ y se dejó enfriar para inocular la cepa.

Previo al experimento, se generó un cultivo madre en 2 matraces de $1000 \mathrm{ml}$ para poder ser usado como inóculo inicial. Los experimentos fueron desarrollados en 2 sets de matraces Erlenmeyer de $100 \mathrm{ml}$, con 3 réplicas cada uno. El primer set fue utilizado para evaluar la tasa de crecimiento y el segundo para analizar la toxicidad de la cepa.
Simultáneamente se prepararon las siguientes proporciones de nitrógeno/fósforo: proporción de nitrógeno alto $\mathrm{N}: \mathrm{P}=24: 1(882 \mu \mathrm{M} / 36,2 \mu \mathrm{M})$, proporción balanceada de nitrógeno $\mathrm{N}: \mathrm{P}=12: 1(441 \mu \mathrm{M} /$ $36,2 \mu \mathrm{M})$ y proporción de nitrógeno limitante $\mathrm{N}: \mathrm{P}=3: 1$ $(110,2 \mu \mathrm{M} / 36,2 \mu \mathrm{M})$.

Cada matraz fue inoculado con una concentración inicial de 250 células $\mathrm{ml}^{-1}$ de A. catenella con cinco réplicas para cada escenario. La duración del experimento fue de un mes, en una sala de cultivo con las siguientes condiciones: temperatura de $15^{\circ} \mathrm{C}$, con un ciclo luz-oscuridad de $14 \mathrm{~h}: 10 \mathrm{~h}$ a $24 \mathrm{\mu Em}^{-2} \mathrm{~s}^{-1} \mathrm{y}$ a una salinidad de 30 PSU. Los recuentos de células fueron realizados cada tres días, extrayendo $1 \mathrm{ml}$ de muestra de cada matraz (con previa agitación suave) en un tubo Eppendorf que contenía 0,5 ml de formalina; posteriormente se llevó el contenido a una placa de conteo Sedgewick-Rafter y se contó la muestra en un microscopio invertido Olympus CK-2.

La tasa de crecimiento específico, $\mu$ (Flynn et al. 1994), fue calculada con la siguiente ecuación:

$$
\mu=\frac{\ln \left(N_{1} / N_{0}\right)}{t_{1}-t_{0}}
$$

donde $\mathrm{N}_{1}$ y $\mathrm{N}_{0}$ son las concentraciones de células en los tiempos $t_{1} y_{0}$.

Los análisis de toxinas fueron efectuados cada seis días y para ello se utilizó la metodología indicada en Frangópulos et al. 2004, la cual consistió en filtrar $20 \mathrm{ml}$ de fitoplancton, con una concentración de células conocida, en un filtro de fibra de vidrio Whatman GF/F de 0,45 $\mu \mathrm{m}$ de tamaño de poro, para luego congelar el filtro en un tubo eppendorf a $-60^{\circ} \mathrm{C}$. Posteriormente el filtro se expuso a ultrasonido en un equipo de modelo ULTRA sonik Ney, y luego se procedió a extraer las toxinas añadiendo $300 \mu \mathrm{l}$ de ácido acético 0,05 $\mathrm{M}$ al material sonicado, y la muestra fue homogenizada usando una punta de pipeta adaptada para ajustarse a la forma del vial. Luego de esto, la muestra fue centrifugada a 14.000 rpm por 10 minutos, después de lo cual $100 \mu \mathrm{L}$ del sobrenadante fueron cuidadosamente extraídos con una micropipeta y transferidos a un vial.

Los análisis de toxicidad fueron realizados por medio de HPLC (cromatografía líquida de alta eficacia), siguiendo el protocolo de Franco \& Fernández (1993) para condiciones isocráticas, con derivatización postcolumna y detección fluorescente 

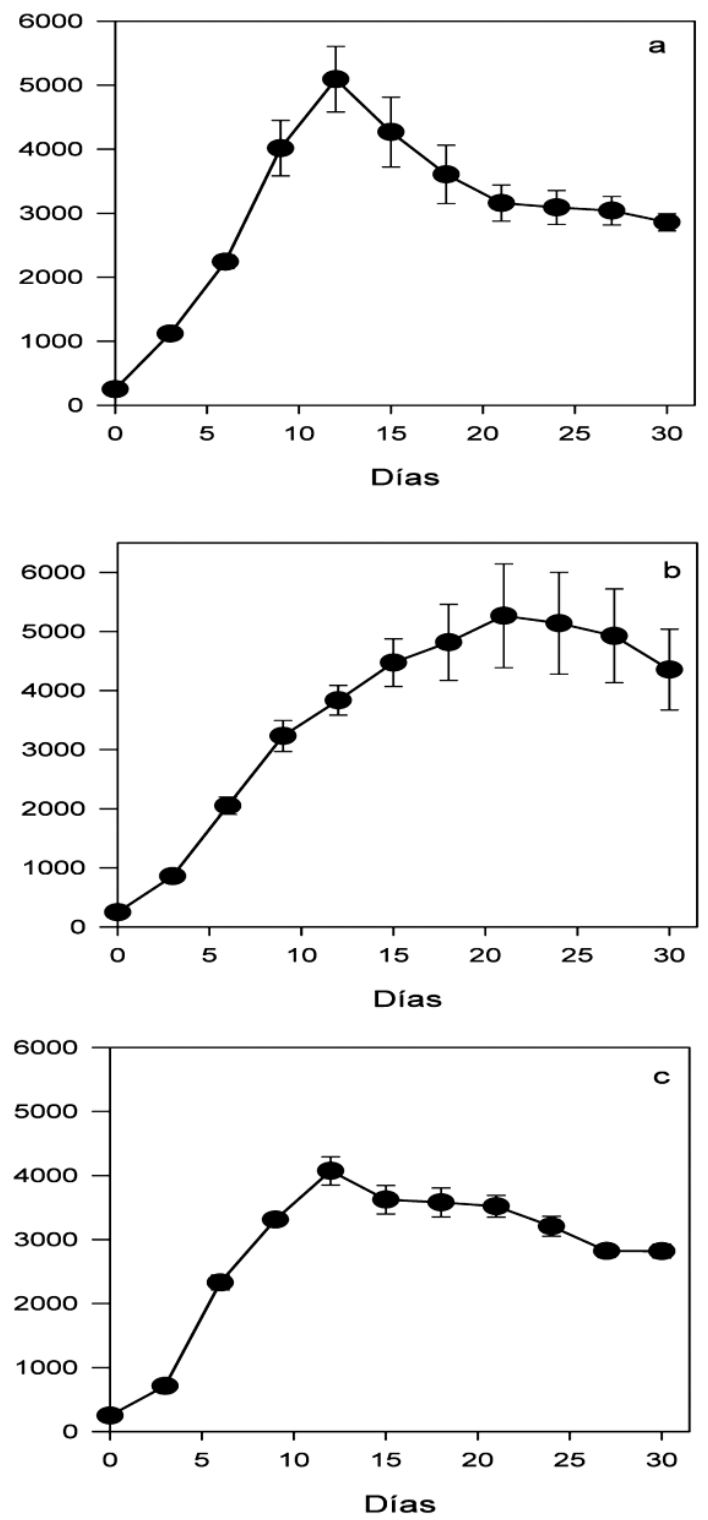

Fig. 1. Abundancia expresada en células $\mathrm{ml}^{-1}$ en (a) tratamiento $\mathrm{N}: \mathrm{P}=24: 1$, (b) $\mathrm{N}: \mathrm{P}=12: 1$, (c) $\mathrm{N}: \mathrm{P}=3: 1$. Los valores se expresan en media \pm E.E.

en línea. Esta información fue registrada directamente en un computador usando una interfase y un programa para Windows, que permite el registro de los cromatogramas. Los perfiles cromatográficos de A. catenella fueron determinados mediante inyecciones de $20 \mu \mathrm{l}$ del extracto de la muestra. Los estándares de toxinas utilizados como referencia fueron suministrados por el National Research
Council de Canadá. En este estudio solamente se cuantificaron las toxinas pertenecientes al grupo de las GTX (GTX 1, 2, 3 y 4).

\section{RESULTADOS}

Proporción de nitrógeno saturado ( $N: P=$ 24:1): La abundancia de Alexandrium catenella registró su máximo valor en el día 12 del experimento, con un total de 5.093 células $\mathrm{ml}^{-1}$. La curva de abundancia nos muestra que la fase lag duró aproximadamente 2 días, y al comienzo del día 3 se puede observar el inicio de la fase exponencial con el valor máximo antes mencionado (Fig. 1a). No se observó una fase estacionaria muy clara, pero si una leve mantención en el número de células desde el día 21 al 27. El menor número de células registradas en este medio una vez iniciado el experimento fue de 2.859 células $\mathrm{ml}^{-1}$ en el día 30 .

Con respecto a la tasa de crecimiento, $A$. catenella en un medio de cultivo saturado de nitrógeno presentó un valor $\mu$ máximo de 0,49 en el día 3 . La tendencia de esta curva es al declive, ya que a medida que transcurrieron los días, las células crecerían menos por el agotamiento de nutrientes (Fig. 2). Así, el valor más bajo fue observado en el día 27 con $\mu=-0,005$. Desde el día 6 hasta el $15(\mu=-0,05)$ se observa una caída brusca en el crecimiento, la cual se nivela a partir de este último día hasta el día 30.

En cuanto al contenido tóxico en este medio de cultivo saturado de nitrógeno, su valor más alto medido fue de 21,28 fmol célula ${ }^{-1}$ registrado en el

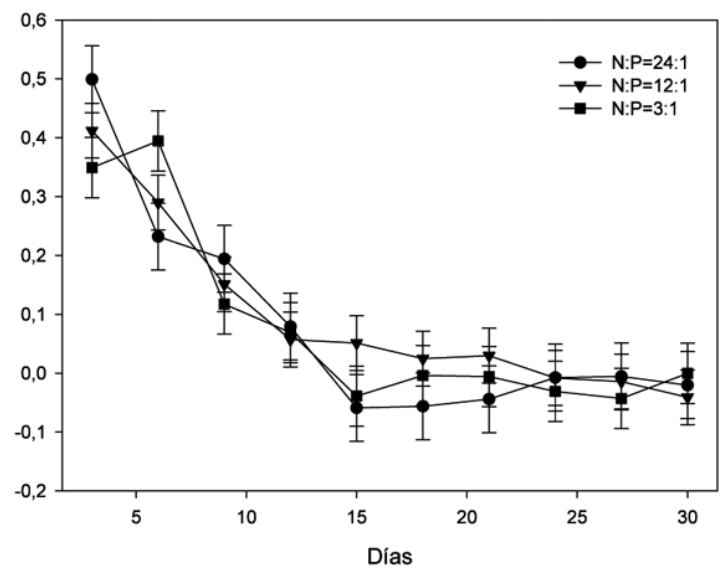

Fig. 2. Tasas de crecimiento específico ( $\mu$ ) en los tres tratamientos estudiados. Los valores se expresan en media \pm E.E. 
día 6 del experimento y que se corresponde con la fase exponencial. Consecuentemente en los días 12 y 18 se observaron valores inferiores que fluctuaron entre los 15 y $17 \mathrm{fmol}$ célula ${ }^{-1}$. Durante la última medición realizada (día 30 ) se registró una toxicidad de 11,12 fmol célula $^{-1}$, valor levemente superior al observado el día que se inició el experimento $(9,04$ fmol célula-1) (Fig. 3).

Con respecto al contenido de toxina específico, se encontraron los 4 tipos de GTX más comunes: GTX1, 2, 3 y 4. En la tabla 1 se aprecia la composición específica de toxinas por día, siendo las de mayor presencia la GTX4 y GTX3. Los mayores valores de toxicidad fueron encontrados día 6 , con rangos entre los 5 y $7 \mathrm{fmol}_{\text {célula }}^{-1}$ para las GTX 1 , 2 y 3 , mientras que la GTX4 con 3,65 fmol célula-1 fue la que presentó la concentración más baja para ese mismo día. Se aprecian proporciones constantes de cada toxina a partir del día 12 al 24, mientras que la toxina GTX4 disminuyó notablemente su concentración en el día 30.

Proporción balanceada de nitrógeno ( $N: P=$ 12:1): La figura $1 \mathrm{~b}$ muestra que la fase lag para la curva de crecimiento dura desde el día 0 hasta el 3 , mientras que la fase exponencial se extendió desde el día 3 hasta el 18, con un valor de abundancia máximo de 4.817 células $\mathrm{ml}^{-1}$.

Luego de este incremento exponencial, se observó una pequeña fase estacionaria donde se mantiene un número relativamente constante de células que varió entre 5.200 y $4.900 \mathrm{ml}^{-1}$. En este tratamiento no se apreció la fase de muerte del cultivo, la que es muy probable que para este caso ocurriría posterior al día 30.

El valor más alto para la tasa de crecimiento de Alexandrium catenella en este tratamiento fue de $0,41 \mu$ en el día 3 , mientras que el más bajo fue de $-0,04 \mu$, indicando nuevamente que la tasa de crecimiento disminuyó a medida que el cultivo crece en el tiempo (Fig. 2).

La toxicidad total de Alexandrium catenella (Fig. 3) mostró un incremento en la fase exponencial, con un valor máximo de $23,02 \mathrm{fmol}$ célula $\mathrm{ml}^{-1}$ (día 6 , ver tabla 7) y a medida que el cultivo se fue acercando a su fase estacionaria la toxicidad disminuyó gradualmente. En el día 30 se registró el valor más bajo con 8,85 fmol célula $\mathrm{ml}^{-1}$.

Proporción de nitrógeno limitante $(N: P=$ 3:1): Este tratamiento mostró una fase lag que se

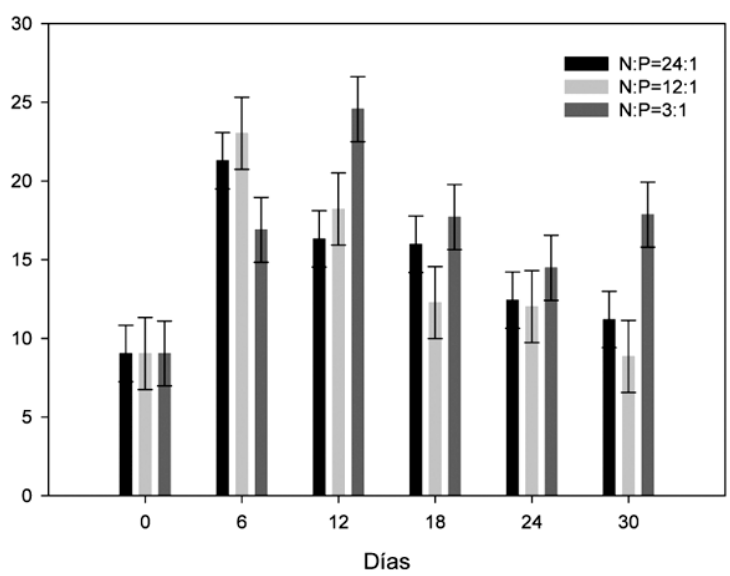

Fig. 3. Toxicidad total para los 3 tratamientos estudiados (fmol célula $^{-1}$ ). Los valores se expresan en media \pm E.E.

extendió durante los primeros 3 días, con un valor máximo de 2.328 células $\mathrm{ml}^{-1}$; mientras que la fase exponencial culminó el día 12 con un valor máximo de 4.072 células $\mathrm{ml}^{-1}$, el más alto registrado para esta concentración durante los 30 días (Fig. 1c). La fase estacionaria se prolongó desde el día 15 hasta el 21 , registrándose posteriormente una leve declinación al final del experimento, con una abundancia al día 30 con 2.818 células $\mathrm{ml}^{-1}$

La fase de muerte tampoco fue distinguible en este tratamiento; a su vez, la tasa de crecimiento para esta concentración no mostró un patrón uniforme, ya que varió considerablemente desde el día 3 al día 15, siendo el mayor crecimiento el día 6 con $\mu=0,39$, mientras que la tasa más baja se midió el día 30 con $\mu=-0,0002$. La curva de crecimiento se estabilizó desde el día 18 del experimento en adelante, manteniéndose el crecimiento de manera constante hasta el día 30 (Fig. 2). El medio limitante de nitratos, obtuvo su mayor valor tóxico el día 12 con $24,56 \mathrm{fmol}_{\text {célula }}{ }^{-1}$ y su menor valor el día inicial del cultivo con 9,04 fmol célula ${ }^{-1}$. Hacia el final del experimento, se registraron $17,86 \mathrm{fmol}_{\text {célula }}{ }^{-1}$. La tendencia general en este tratamiento fue de un incremento de la toxicidad en los primeros 12 días y una disminución a contar del día 12 al 24, mientras que en el día 30 se incrementó levemente el contenido tóxico celular (Fig. 3).

La composición de las GTXs muestra que la GTX3 presentó el valor más alto en todo el cultivo con 11,19 fmol célula ${ }^{-1}$ en el día 12 , equivalentemente a un $43 \%$ de total de las toxinas para ese 
Tabla 1. Composición específica de toxinas correspondientes a los tres tratamientos estudiados durante los 30 días de experimento. Los valores se expresan en media \pm E.E.

\begin{tabular}{cccccccc}
\hline Tratamiento & Días/toxina & GTX4 & GTX1 & GTX3 & GTX2 & Total & E.E. \\
\hline $\mathrm{N}: \mathrm{P}=24: 1$ & 0 & 4,18 & 1,31 & 2,31 & 1,23 & 9,04 & 0,69 \\
& 6 & 3,65 & 5,52 & 6,36 & 5,75 & 21,28 & 0,58 \\
& 12 & 3,97 & 2,55 & 6,74 & 3,05 & 16,32 & 0,93 \\
& 18 & 4,09 & 3,92 & 4,19 & 3,78 & 15,98 & 0,09 \\
& 24 & 3,67 & 3,92 & 3,76 & 4,05 & 15,40 & 0,09 \\
& 30 & 1,15 & 3,92 & 2,10 & 4,03 & 11,19 & 0,71 \\
\hline $\mathrm{N}: \mathrm{P}=12: 1$ & 0 & 4,18 & 1,31 & 2,31 & 1,23 & 9,04 & 0,69 \\
& 6 & 4,08 & 5,79 & 7,05 & 6,10 & 23,02 & 0,62 \\
& 12 & 4,48 & 3,47 & 6,46 & 3,80 & 18,21 & 0,67 \\
& 18 & 3,73 & 2,96 & 2,85 & 2,73 & 12,27 & 0,23 \\
& 24 & 3,68 & 2,51 & 3,25 & 2,57 & 12,01 & 0,28 \\
$\mathrm{~N}: \mathrm{P}=3: 1$ & 30 & 1,97 & 2,68 & 1,53 & 2,67 & 8,85 & 0,28 \\
& 0 & 4,18 & 1,31 & 2,31 & 1,23 & 9,04 & 0,69 \\
& 6 & 2,30 & 4,82 & 4,61 & 5,18 & 16,89 & 0,65 \\
& 12 & 6,06 & 3,29 & 11,19 & 4,01 & 24,56 & 1,78 \\
& 18 & 5,66 & 4,16 & 4,18 & 3,70 & 17,70 & 0,43 \\
& 24 & 4,49 & 3,50 & 2,90 & 3,59 & 14,47 & 0,33 \\
& 30 & 5,91 & 4,70 & 2,94 & 4,31 & 17,86 & 0,61 \\
\hline
\end{tabular}

día. Para la GTX4, el valor observado en el día 0 (4,18 fmol célula-1), a pesar de no ser el más alto para esta concentración, si fue el mayor valor de total de toxinas detectadas. Finalmente en el caso de la GTX1 y 2, los valores de toxicidad observados fueron similares y constantes con respecto a los de GTX 4 y 3, manteniendo su cantidad porcentual en el tiempo. Para el último día del experimento, la tendencia observada indicó una baja cantidad de toxinas así como también porcentajes relativamente constantes entre los 4 tipos de GTX analizadas. La mayor cantidad de toxinas fueron medidas en este medio limitante de Nitratos (Tabla 1).

Los análisis de varianza realizados mostraron que no existieron diferencias significativas $(p>0,05)$ entre las abundancias de Alexandrium catenella de los tres tratamientos estudiados. Con respecto a las tasas de crecimiento, estas mostraron que aunque los valores de crecimientos varían entre los tratamientos, tampoco existieron diferencias significativas ( $p>0,05)$. La tendencia de las toxinas totales en los tres tratamientos es similar; en este caso también se efectuó un análisis de varianza el cual arrojó que no existieron diferencias significativas entre las toxinas de los tres tratamientos ( $p>0,05)$ (Fig. 3). No obstante, las toxicidades se incrementan en la fase exponencial y decaen al final del cultivo pero en diferentes días y cantidad, en las 3 proporciones (tabla 1).

\section{DISCUSIÓN}

Los resultados obtenidos indican que los valores de abundancia estuvieron relacionados de manera inversa con la tasa de crecimiento, luego, a medida que hay mayor número de células en el cultivo, la rapidez con la cual estas se dividirán en términos de reproducción será cada vez más menor. Navarro et al. (2006) encontraron en condiciones similares de cultivo en $A$. catenella, 6.389 células $\mathrm{ml}^{-1}$ como el mayor valor recién después del día 50 de su estudio, mientras que en este estudio el valor más alto observado fue al día 21 con 5.266 células $\mathrm{ml}^{-1}$. Esta diferencia en la abundancia puede atribuirse a que estos autores utilizaron una cepa diferente a la utilizada en este estudio; además de las diferencias registradas para la mayor cantidad de intensidad lumínica y diferente temperatura de crecimiento $\left(16^{\circ} \mathrm{C}\right)$, parámetros que se encuentran entre los más importantes a la hora de estimular el crecimiento de las microalgas. Frangópulos et al. 2004 registraron en el día 15 de cultivo para A. tamarense, A. minutum y A. andersonii los siguientes valores: 2.500, 15.000 y 9.000 células $\mathrm{ml}^{-1}$, respectivamente. Al comparar ambos estudios, el valor más alto de $A$. catenella obtenido para el mismo día fue de 4.472 células $\mathrm{ml}^{-1}$, lo que deja en evidencia las grandes diferencias existentes entre 
especies de un mismo género, cuando nos referimos a la abundancia. Esto se debe a que en la temperatura utilizada en nuestro estudio $\left(15^{\circ} \mathrm{C}\right)$, estas especies la aprovecharían de mejor manera para generar cambios en su maquinaria metabólica que se traduciría en incrementos en las tasas de división por tiempo, como lo señala Navarro et al. (2006).

El crecimiento de esta especie bajo las condiciones de cultivo establecidas varió en los tres tratamientos analizados, pero siempre en el mismo orden de magnitud. El mayor valor se encontró en la concentración $\mathrm{N}: \mathrm{P}=(24: 1)$ con una tasa de crecimiento de $0,50 \mu$, lo que concuerda con lo observado en otros estudios similares (Wang et al. (2005) para $A$. tamarense; Lim et al. (2009) para A. minutum), en la fase exponencial. El crecimiento de A. catenella en la concentración experimental $\mathrm{N}: \mathrm{P}=12: 1$ mostró una tendencia muy similar a la observada en el escenario anterior, posiblemente debido a que en el término de la fase lag de crecimiento e inicio de la fase exponencial, la absorción de nutrientes por estos dinoflagelados nunca va a ser mayor, aunque posean concentraciones más altas de nutrientes en el medio, ya que su fisiología y/o metabolismo interno no lo permite (Anderson et al. 2002). En cambio en el medio limitante de nitratos ( $\mathrm{N}: \mathrm{P}=3: 1)$, el crecimiento fue menor, tal y como fue observado en para este estudio y que coinciden con lo expuesto en estudios previos por Wang et al. (2005) para A. tamarense, el cual tuvo un bajo crecimiento en similares condiciones de cultivo.

Estas estrategias de las microalgas corresponden a respuestas ecológicas para distintos fines. Previamente se mencionó que el crecimiento es más alto con mayor cantidad de nitratos en el medio, pero sólo hasta llegar a cierto punto, en donde el alga deja de utilizarlo para crecer, ya que no puede sintetizar más, y lo almacena con la finalidad de ocuparlo para próximas divisiones celulares (Collos et al. 2004), lo que se puede interpretar como una estrategia para resguardar nutrientes en caso de que se agoten en el medio.

En este estudio, las toxinas totales no presentaron diferencias significativas entre tratamientos utilizados durante el desarrollo experimental; sin embargo, en la concentración limitante de nitrato se cuantificó la mayor cantidad de toxinas totales, lo cual es aceptado de manera general como una tendencia en este tipo de experimentos (Choua et al., 2004; Hwang \& Lu, 2000; Lim et al., 2006; Maestrini et al. 1999, Murata et al., 2006). Flynn et al. (1994) comprobaron que el fósforo está implicado en la regulación del metabolismo de las toxinas en $A$. tamarense, y similar resultado fue corroborado para A. minutum por Lim et al. (2009), por lo cual no sería raro encontrar en radios donde el nitrógeno sea menor y el fósforo mayor, en proporción, un aumento en las toxinas totales y una composición tóxica similar, independiente de la especie de Alexandrium con la que se esté trabajando. En este estudio, se analizaron el grupo de toxinas correspondientes a las gonyaulatoxinas, denominadas comúnmente GTX; para los tres tratamientos analizados, donde la toxina que tuvo la mayor concentración registrada fue la GTX3, correspondientemente, la mayor concentración de esta toxina se encontró en el tratamiento $\mathrm{N}: \mathrm{P}(3: 1)$, por lo que se podría asumir que esta tendencia fuese general para todo el género Alexandrium. Al respecto, Anderson (1997) reconoció que la limitación de fósforo aumenta la producción de GTX2 y GTX3, y que la limitación de nitrógeno ayuda a la producción de C1, C2, GTX1 y GTX4, lo cual sería cierto para un proporción de $\mathrm{N}$ y $\mathrm{P}$ muy bajo, situación que no ocurrió en el caso de este estudio, en donde la diferencia entre estas proporciones fue menor. La información existente sobre las toxinas totales y su composición específica es muy variable entre especies del mismo género y también entre cepas de la misma especie (Chou et al. 2004; Leong et al., 2004; Lim et al., 2006). Otros autores como Compagnon et al. (1998) señalan que las toxinas de un cultivo de A. catenella contrastadas con las toxinas encontradas en choritos no son equivalentes, en cuanto a cantidad y composición específica. Todo lo anterior dificulta mucho el dilucidar una tendencia para la composición específica de toxinas en cultivos de organismos fitoplanctónicos.

Se puede afirmar después de lo expuesto que el estudio de las toxinas en dinoflagelados es un fenómeno complejo. No obstante, hay ciertas líneas de investigación actuales que postulan que la composición tóxica es una característica bioquímica estable para cada dinoflagelado y que esto puede utilizarse como una huella genética para análisis cromotaxonómicos para diferenciar poblaciones (Wang et al. 2006). 
La contraparte de esto es que existen publicaciones que señalan que realizar análisis de este tipo no sería lo más indicado, ya que como se ha mencionado anteriormente, la toxicidad de Alexandrium varía bastante y por múltiples factores. Esto tiene mucho sentido, ya que numerosos estudios genéticos y de composición tóxica de este género, han encontrado que algunas cepas de especies de Alexandrium cambian su toxicidad dependiendo del origen biogeográfico de la misma (Choua et al., 2004; Leong et al., 2004; Lim et al. 2006), lo que conllevaría a que algunas especies generen toxinas en un área del globo y que la misma especie en otra parte no sea tóxica, como ocurre con A. tamarense (Yentsch, 1978, Hallegraef, 1991, Moestrup \& Hansen, 1988, Fukuyo et al. 1988).

Actualmente, la información disponible indica que en el caso de A. catenella, esta especie es tóxica en sus diferentes cepas en prácticamente todo el globo, pero presenta diferencias en la composición de toxinas de las mismas. Sfeir et al. (2005) en un estudio realizado en Chile, trabajaron con 5 cepas de la región de los Lagos y Aysén, encontrando variaciones en la composición específica de las cepas y la toxicidad total de estas mismas, con valores entre los 41,41 fmol célula-1 (cepa ACC01) y 235,95 fmol célula ${ }^{-1}$ (cepa ACSD01). Estos valores son más altos comparados con los obtenidos en este estudio, probablemente sustentados en que la duración de su estudio fue mayor y en la variación inherente que ya se mencionó que existe en la composición y cantidad de toxinas entre una misma especie.

El análisis de varianza para los resultados obtenidos no mostró diferencias significativas entre los datos de abundancia, crecimiento y toxicidad $(p>0,05)$, probablemente debido a que si bien estos datos tienen diferencias entre ellos, las mismas son muy pequeñas. Para que estas diferencias fueran significativas, se recomienda usar radios de nitratos y fosfatos en diferentes órdenes de magnitud, como los radios utilizados por, Bechemin et al. (1999), para A. minutum, Hu et al. (2006) y Lim et al. (2009) para A. tamarense. Estos autores utilizaron entre 5 y 6 tipos diferentes de proporciones N y P con valores de 500, 300, 100, 30 . Eventualmente, con estos radios tan distintos, la significancia de los datos de crecimiento, abundancia y toxicidad sería mucho mayor.

Recientemente Aguilera-Belmonte et al. 2011 trabajaron con 7 cepas chilenas, de A. catenella; en todas se observó un crecimiento y abundancia superior a los escenarios utilizados en este estudio, lo cual puede deberse a la cantidad de inoculo inicial que utilizaron, i.e. al día 5 de experimento sus cultivos registraban más de 5.000 células $\mathrm{ml}^{-1}$, lo cual es muy superior a lo observado al mismo día de este estudio y considerando también que 5.000 células $\mathrm{ml}^{-1}$ fue la cantidad máxima en este trabajo. En cuanto a las toxinas, su mayor valor fue de 96,9 fmol célula ${ }^{-1}$, mientras que en este estudio fue de 24,56 fmol célula ${ }^{-1}$, lo cual puede estar relacionado con lo previamente mencionado y a que estos autores utilizaron el medio L1 con las proporciones iniciales diferentes a las usadas para este experimento; adicionalmente su gran abundancia de células también puede generar mayor cantidad de toxinas de igual modo como ocurre en una floración en la naturaleza (Anderson et al. 2012).

En el futuro el estudio del cuociente N:P en dinoflagelados tóxicos debería ser considerado como una herramienta esencial para el desarrollo de una línea de base de estudios autoecológicos, ya que esto determina las condiciones óptimas de nutrientes para estos organismos y de esta manera se puede lograr una aproximación diferente para poder predecir el comportamiento de las floraciones tóxicas a nivel local y mundial.

\section{AGRADECIMIENTOS}

Se agradece al Instituto de Fomento Pesquero (IFOP) Base Zonal Punta Arenas, por facilitar las instalaciones para poder realizar el presente estudio, especialmente a Claudia Zamora por los análisis de toxinas realizados por HPLC. Al Dr. Daniel Varela, de la Universidad de Los Lagos, encargado del cepario del centro i-mar, quién cedió gentilmente la cepa AY2 de Alexandrium catenella, con la cual se llevó a cabo este trabajo.

El presente estudio fue financiado en su totalidad por el proyecto FONDECYT regular №1080548.

\section{LITERATURA CITADA}

Aguilera-Belmonte, A., I. Inostroza, J.M., Franco, P. Riobó \& P.I. Gómez 2011. The growth, toxicity and genetic characterization of seven strains of Alexandrium catenella (Whedon and Kofoid) Balech 1985 (Dinophyceae) isolated 
during the 2009 summer outbreak in southern Chile, Harmful Algae, 12: 105-112.

Anderson, D.M., D.M. Kulis, J.J. Sullivan, S. Hall, C. Lee 1990. Dynamics and physiology of saxitoxin production by the dinoflagellates Alexandrium spp. Marine Biology, 104: 511-524.

Anderson, D.M. 1998. Physiology and bloom dynamics of toxic Alexandrium species, with emphasis on life cycle transitions. In: Anderson D. M., A.D. Cembella and G. M. Hallegraeff, (eds.) Physiological Ecology of Harmful Algae Blooms. NATO ASI Series, G 41: 29-48.

Anderson, D.M. (1984). Shellifish toxicity and dormant cysts in toxic dinoflagellate blooms. En: Ragelis, E.P. (ed.): Seafood toxins. ACS Symposium Series 262, American Chemical Society, Washington, DC, pp. 125-138.

Anderson, D. 1997. "Turning back the harmful red tide". Nature, 388: 513-514.

Anderson, D., P. Glibert \& J. Burkholder 2002. Harmful algal blooms and Eutrophication: Nutrient Sources, composition and consequences. Estuaries, 25(4b): 704-726.

Anderson, D. M., D. Cembella \& G.M. Hallegraef 2012. Progress in Understanding Harmful Algal Blooms: Paradigm Shifts and New Technologies for Research, Monitoring, and Management. Annual Review of Marine Science, 4:143-76.

Balech E. 1985. The genus Alexandrium or Gonyaulax of the tamarensis group. In: Toxic Dinoflagellates (Ed. by D.M. Anderson, A.W. White \& D.G. Baden), pp. 33-38. Elsevier, New York.

Béchemin, C., D. Grzebyk, F. Hachame, C. Hummert \& S. Maestrini 1999.Effect of different nitrogen ratios on the toxin content in A.minutum. Aquatic Microbial Ecology, 20: 157-165.

Boyer, G.L., J.J. Sullivan, R.J. Andersen, P.J Harrison \& F.J.R. Taylor 1987. Effect of nutrient limitation on toxin production and composition in the marine dinoflagellate Protogonyaulax tamarense. Marine Biology, 96: 123-128

Burkholder, J.M., P.M. Glibert \& H.M. Skelton 2008. Mixotrophy, a major mode of nutrition for harmful algal species in eutrophic waters. Harmful Algae, 8: 77-93.
Cembella, Ad., 1998. Ecophysiology and metabolism of paralytic shellfish toxins in marine microalgae. En: Anderson, D, M., Cembella, A.D., Hallegraef, G.M (Eds), Physiological Ecology of Harmful Blooms. Springer, Heidelberg, pp.381-403.

Collos, Y., C. Gagne, M. Laabir, A. Vaquer, P. Cecchi \& P. Souchu 2004. Nitrogenous nutrition of Alexandrium catenella (Dinophyceae) in cultures and in Thau Lagoon, Southern France. France Journal of Phycology, 40: 96-103.

Collos, Y., A. Vaquer, M. Laabir, E. Abadie, T. Laugier, A. Pastoureaud \& P. Souchu 2007. Contribution of several nitrogen sources to growth of Alexandrium catenella during blooms in Thau lagoon, Southern France. Harmful Algae, 6: 781-789.

Collos, Y., B. Bec, C. Jauzein, E. Abadie., T. Laugier, L. Jacques, A. Pastoureaud, P. Souchuand \& A. Vaque 2009 Oligotrophication and emergence of picocyanobacteria and a toxic dinoflagellate in Thau lagoon, southern France. Journal of Sea Research, 61: 68-75.

Chee, S., Y. Leong, Ai. Murata, Y. Nagashima \& S. Taguchi 2004. Variability in toxicity of the dinoflagellate Alexandrium tamarense in response to different nitrogen sources and concentrations. Toxicon, 43:407-415.

Choua, H.N., Y.M. Chen \& C.Y. Chen 2004. Variety of PSP toxins in four culture strains of Alexandrium minutum collected from southern Taiwan. Toxicon, 43: 337-340.

Compagnon, D., G. Lembeye, N. Marcos, N. RuizTagle \& N. Lagos 1998. Accumulation of paralytic shellfish poisoning toxins in the bivalve Aulacomya ater and two carnivorous gastropods Concholepas concholepas and Argobuccinum ranelliformes during an Alexandrium catenella bloom in southern Chile. Journal of Shellfish Research, 17: 67-73.

Flynn, K., J.M. Franco, P. Fernández, B. Reguera, M. Zapata, G. Wood \& K.J. Flynn 1994. Changes in toxin content, biomass and pigments of the dinoflagellate Alexandrium minutum during nitrogen refeeding and growth into nitrogen or phosphorus stress. Marine Ecology Progress Series, 111: 99-109.

Franco, J.M \& P. Fernández 1993. Separation of PSP toxins by reversed phase high performan- 
ce liquid chromatography, with postcolumn reaction and fluorimetric detection. Chromatographia, 35: 613-620.

Frangópulos, M., C. Guisande, E. deblas \& I. Maneiro 2004. Toxin production and competitive abilities under phosphorus limitation of Alexandrium species. Harmful Algae, 3(2): 131-139.

Fukuyo, Y., P. Pholpunthin \& K. Yoshida 1988. Protogonyaulax (Dinophyceae) in the Gulf of Thailand. Bulletin of Plankton Society of Japan, 35: 9-20.

Glibert, P., D. Anderson, P. Gentien, E. Graneli \& K. Sellner 2005. The global complex Phenomena of Harmful Algal blooms. Oceanography, 18(2): 134-135.

Guillard, R. \& P. Hargraves 1993. Stichochrysis immobilis is a diatom, not a chrysophyte. Phycologia, 32: 234-236.

Guzmán, L., H. Pacheco, G. Pizarro \& C. Alarcón 2002. Alexandrium catenella y veneno paralizante de los mariscos en Chile. En: floraciones algales nocivas en el cono sur americano Sar, E.A., M.E. Ferrario \& B. Reguera Instituto Español de Oceanografía, 2002.

Hallegraeff, G.M., C.J. Bolch, S.I. Blackburn \& Y. Oshima 1991. Species of the toxigenic dinoflagellate genus Alexandrium in southeastern Australian waters. Botanica Marina, 34: 575-587.

Hallegraeff, G.M. (1995). Harmful algal blooms: a global overview. En: Hallegraeff, G.M., Anderson, D.M. y Cembella, A.D. (eds): Manual on Harmful Marine Microalgae. IOC Manuals and Guides № 33, UNESCO, pp. 1-22.

Hansen, G. \& E. Moestrup 1998. Fine-structural characterization of Alexandrium catenella (Dinophyceae) with special emphasis on the Flagellar apparatus. European Journal of Phycology, 33: 281-291.

$\mathrm{Hu}, \mathrm{H}$., W. Chen, Y. Shi \& W. Cong 2006. Nitrate and phosphate supplementation to increase toxin production by the marine dinoflagellate A.tamarense. Marine Pollution Bulletin, 52: 756-760.

Hwang, D.F \& Y.H. Lu 2000. Infuence of environmental and nutritional factors on growth, toxicity, and toxin profile of dinoflagellate Alexandrium minutum. Toxicon, 38 1491-1503.
Jauzein, C., C. Labry, Á. Youenou, J. Quéré, D. Delmas \& Y. Collos 2010. Growth and phosphorus uptake by the toxic dinoflagellate Alexandrium catenella (Dinophyceae) in response to phosphate limitation. Journal of Phycology, 46: 926-936.

Jeong, HJ., J.Y. Park , J.H. Nho, M.O. Park, J.H. Ha, K.A. Seong, C. Jeng, C.N Seong \& K.Y. Yih 2005. Feeding by red-tide dinoflagellates on the cyanobacterium Synechococcus. Aquatic Microbial Ecology, 41:131-143.

Johansson, N \& E. Granéli 1999. The influence of different N:P supply ratios on cell density, chemical composition and toxicity of Prymnesium parvum (Haptophyta) in semi-continuous cultures. Journal of Experimental Marine Biology Ecology, 139: 243-258

John, E.H. \& K.J. Flynn 2000. Growth dynamics and toxicity of Alexandrium fundyense (Dinophyceae): the effect of changing N:P supply ratios on internal toxin and nutrient levels. European Journal of Phycology, 35: 11-23

Leong, S., A. Murata, Y. Nagashima \& S. Taguchi 2004. Variability in toxiciyty of the dinoflagellate A.tamarense in response to different nitrogen sources and concentrations.Toxicon, 43: 407-415.

Lilly, E.L., K.M. Halanych, \& D.M. Anderson 2007. Species boundaries and global biogeography of the Alexandrium tamarense complex (Dinophyceae). Journal of Phycology, 43: 1329-1338.

Lim, P.T \& T. Ogata 2005. Salinity effect on growth and toxin production of four tropical Alexandrium species (Dinophyceae). Toxicon, 45: 699-710.

Lim, P.T., C. Leaw, G. Usuo, A. Kobiyama, K. Koike \& T. Ogata 2006. Effects of light and temperature on growth, nitrate uptake, and toxin production of two tropical dinoflagellates: Alexandrium tamiyavanichii and Alexandrium minutum (Dinophyceae). Journal of Phycology, 42: 786-799.

Lim, P.T., C.P. Leaw \& A. Kobiyama 2009. Growth and toxin production of tropical Alexandrium minutum Halim (Dinophyceae) under various nitrogen to phosphorus ratios. Journal of Applied Phycology: DOI 10.1007/s10811009-9443-8. 
Maestrini, S., C. Bechemin, D. Grzebyk \& C. Hummert 1999. Phosphorus limitation might promote more toxin content in the marine invader dinoflagellate Alexandrium minutum. Plankton Biology and Ecology, 47 (1): 7-11.

Matsuoka, K. \& Y. Fukuyo 2000. Technical guida for Modern Dinoflagellate cyst study. Pag. 9-11. Definition of cyst, WESTPACHAB/ WESTPAC/IOC 2000.

Moestrup, O. \& P.J. Hansen 1988. On the occurrence of the potentially toxic dinoflagellates Alexandrium tamarense (= Gonyaulax excavata) and A. ostenfeldii in Danish and Faroese waters. Ophelia, 28: 195-213.

Murata, Ai., S. Chee, Y. Leong, Y. Nagashima \& S. Taguchi 2006. Nitrogen:Phosphorus supply ratio may control the protein and total toxin of dinoflagellate Alexandrium tamarense. Toxicon, 48: 683-689.

Navarro, J.M., M.G. Muñoz \& A.M. Contreras 2006. Temperature as a factor regulating growth and toxin content in the dinoflagellate Alexandrium catenella. Harmful Algae, 5: 762-769.

Seong, KA., H.J. Jeong, S. Kim, G.H. Kim, J.H. Kang 2006. Bacterivory by co-occurring red- tide algae, heterotrophic nanoflagellates, and ciliates on marine bacteria. Marine Ecology Progress Series, 322: 85-97.

Sfeir, A., M. Seguel \& F. Navarrete 2005. Composición toxicológica de distintas cepas Chilenas de A.catenella. En: libro de resumenes XXV Congreso de Ciencias del Mar.

Shimizu, Y. 1996. Microalgal metabolites: a new perspective. Annual Review Microbiology, 50: 431-465.

Wang, D.Z. \& D. Hsieh 2005. Growth and toxin production in batch cultures of a marine dinoflagellate Alexandrium tamarense HK9301 isolated from the South China Sea. Harmful Algae, 4: 401-410.

Whedon, W.F. \& C.A. Kofoid 1936. Dinoflagellates of the San Francisco region. I. On the skeletal morphology of two new species, Gonyaulax catenella and G. acatenella. University of California Publications in Zoology, 41: 25-34.

Yentsch, C.M., B. Dale \& J.W. Hurst 1978. Coexistence of toxic and nontoxic dinoflagellates resembling Gonyaulax tamarensis in New England coastal waters (NW Atlantic). Journal of Phycology, 14: 330-332. 
